\title{
An Upper Bound for the Ground-State Energy of an Antiferromagnetic Chain with Next-Nearest-Neighbor Interaction
}

\author{
H. P. van de Braak, ${ }^{1}$ W. J. Caspers, ${ }^{1}$ P. K. H. Gragert, ${ }^{1}$ \\ and M. W. M. Willemse ${ }^{1}$
}

Received September 27, 1978

\begin{abstract}
A trial function for the ground state of the antiferromagnetic linear chain leads to an equation for an upper bound of the ground-state energy per spin. This equation is solved exactly.
\end{abstract}

KEY WORDS: Antiferromagnetism; magnetic ordering; ground-state energy; linear chain; renormalization.

\section{INTRODUCTION}

In a previous paper ${ }^{(1)}$ (henceforth referred to as I), an approximate value of the ground-state energy per spin of a linear antiferromagnetic system was determined with a renormalization procedure. In the present work we consider the same system, but now an upper bound of the ground-state energy is found with a trial function, renormalization techniques leading to a functional equation of a special type. Other applications of renormalization techniques for quantum spin systems can be found in the papers given in Ref. 2.

The interaction in our model is of the Heisenberg type, coupling neighbor spins and next-nearest neighbors. We only consider isotropic interactions, but our method may be readily applied to all kinds of spin systems. Restriction to spins $S=\frac{1}{2}$ is essential for our method.

Again the system is divided into cells of three spins, as was done in I, and again we consider the product state of the ground states of the different cells as our zeroth-order ground state. So far our new approach is identical

\footnotetext{
${ }^{1}$ Twente University of Technology, Enschede, The Netherlands.
} 
to the one exposed in I. A recapitulation of the essential steps is found in Section 2 of this paper.

In Section 3 a unitary operator is introduced, containing a variational parameter, which, for an appropriate choice of the value of the parameter, will improve the ground state. Instead of transforming the ground state we may, equivalently, transform the Hamiltonian. Projecting the transformed Hamiltonian onto the manifold of the zeroth-order ground state, a reproduction of the original Hamiltonian is found, in the same way as in I. This reproduction leads to a functional equation for an upper bound of the groundstate energy per spin, the equation connecting values of this energy for different $\gamma$, the ratio between next-nearest-neighbor and nearest-neighbor interaction constants. The functional equation also contains the variational parameter, for which an optimal value should be found. Section 4 is devoted to the derivation of the equation on the basis of the scaling procedure in Section 3.

Having derived the functional equation, we have reduced the problem to a purely mathematical one, which is solved exactly in Section 5 . In the first instance a solution was computed by an iteration process. The numerical solution suggested an analytic one, which was found indeed. We could only prove, however, that this solution corresponds to a relative minimum, whereas we believe that our choice of the variational parameter corresponds to an absolute minimum. In Section 6 a short discussion of our results is given and a comparison with other results for the same problem is made. ${ }^{(4-7)}$

\section{HAMILTONIAN AND ZEROTH-ORDER GROUND STATE}

We summarize the division of the Hamiltonian of the chain into a part corresponding to internal interactions in the cells of three spins and a part representing the interaction between cells. As was stressed in I, renormalization methods may also be generalized by taking cells of a larger odd number of spins.

For the case of cells of three spins we have

$$
\begin{aligned}
H & =4 \sum_{i} \mathbf{S}_{i} \cdot \mathbf{S}_{i+1}+4 \gamma \sum_{i} \mathbf{S}_{i} \cdot \mathbf{S}_{i+2} \\
H & =H_{0}+H^{\prime} \\
H_{0} & =\sum_{k} H_{0, k}, \\
H_{0, k} & \left.=4\left[\mathbf{S}_{3 k-1} \cdot \mathbf{S}_{3 k}+\mathbf{S}_{3 k} \cdot \mathbf{S}_{3 k+1}\right)+\gamma \mathbf{S}_{3 k-1} \cdot \mathbf{S}_{3 k+1}\right] \\
H^{\prime} & =\sum_{k} H_{k, k+1}^{\prime}, \\
H_{k, k+1}^{\prime} & =4\left[\mathbf{S}_{3 k+1} \cdot \mathbf{S}_{3 k+2}+\gamma\left(\mathbf{S}_{3 k} \cdot \mathbf{S}_{3 k+2}+\mathbf{S}_{3 k+1} \cdot \mathbf{S}_{3 k+3}\right)\right]
\end{aligned}
$$

In (1) the constant $\gamma$ measures the relative strength of the next-nearest- 
neighbor interaction, the nearest-neighbor interaction being normalized in a convenient way. The part $H_{0}$ corresponds to internal interactions in the cells, whereas $H^{\prime}$ gives the coupling between cells.

The ground state of the zeroth-order part of the Hamiltonian $H_{0}$ is a product of doublet states for each individual cell, as was discussed at length in $I$, in which the symbols used here are fully explained:

$$
\begin{aligned}
\left|0, m_{k}\right\rangle & =\prod_{k}\left|\frac{1}{2}+, m_{k}\right\rangle, \quad m_{k}= \pm \frac{1}{2} \\
H_{0, k}\left|\frac{1}{2}+, m_{k}\right\rangle & =\epsilon_{0}(\gamma)\left|\frac{1}{2}+, m_{k}\right\rangle, \quad \epsilon_{0}(\gamma)=-4+\gamma
\end{aligned}
$$

The solution of the zeroth-order secular problem is written in a compact form by the formula

$$
H_{0}\left|0, m_{k}\right\rangle=\frac{1}{3} N \epsilon_{0}(\gamma)\left|0, m_{k}\right\rangle
$$

in which $N$ stands for the total number of spins, which is considered to be large $(N \rightarrow \infty)$. In (3), N/3 equals the number of cells. The next section is devoted to the introduction of a suitable unitary transformation, after which the transformed Hamiltonian may be projected to give a reproduction of the original Hamiltonian in the form of a Hamiltonian for cell spins.

\section{VARIATIONAL OPERATORS AND PROJECTION OF THE HAMILTONIAN}

We now introduce a suitable unitary transformation, which depends on a real parameter. This unitary operator may be considered to transform the highly degenerate ground state. The total Hamiltonian (1) may be projected onto this transformed set, the projection resulting in a reproduction of the original secular problem, the original spins being replaced by cell spins $\left(S^{\prime}=\frac{1}{2}\right)$, corresponding to the Kramers doublets introduced in (2). The projection can be optimized by choosing the right value for the parameter, as will be explained in Section 4.

In the following, however, we shall use a procedure that is mathematically equivalent. We first transform the Hamiltonian and then project onto the original set (2).

The unitary operator is of a rather simple structure, being a product of terms pertaining to two adjacent cells, all terms commuting with one another

$$
\begin{aligned}
u(a) & =\prod_{k} u(k, a) \\
u(k, a) & =\exp \left[i 2 a\left(\mathbf{S}_{3 k+1} \cdot \mathbf{S}_{3 k+2}+\frac{1}{4}\right)\right]=\exp [i a P(3 k+1,3 k+2)]
\end{aligned}
$$


The operators $P$ are well-known spin-exchange operators ${ }^{(3)}$ with the properties

$$
\begin{aligned}
& P^{-1}(1,2)=P^{+}(1,2)=P(1,2) P_{12} \\
& P_{12} \mathbf{S}_{1} P_{12}=\mathbf{S}_{2}, \quad P_{12} \mathbf{S}_{2} P_{12}=\mathbf{S}_{1} \\
& {\left[\mathbf{S}_{1}, P_{12}\right]=-\left[\mathbf{S}_{2}, P_{12}\right]=-2 i\left(\mathbf{S}_{1} \times \mathbf{S}_{2}\right)}
\end{aligned}
$$

from which follows in an easy way

$$
\begin{aligned}
& u(k, a)=\cos a+2 i\left(\mathbf{S}_{3 k+1} \cdot \mathbf{S}_{3 k+2}+\frac{1}{4}\right) \sin a \\
& {\left[u(a), \mathbf{S}^{2}\right]=0, \quad \mathbf{S}=\sum_{i} \mathbf{S}_{i} .}
\end{aligned}
$$

It should further be stressed that $u(a)$ has the full translational symmetry of the lattice of cells.

The zeroth-order projection operator, projecting onto the set $\left|0, m_{k}\right\rangle$ of (2), may be written as follows:

$$
P_{0}=\sum_{m_{k}= \pm 1 / 2}\left|0, m_{k}\right\rangle\left\langle 0, m_{k}\right|
$$

It projects onto the space corresponding to the lowest eigenvalue of $H_{0}$, which has dimension $2^{N / 3}$. The projection of $H_{0}$ is

$$
P_{0} H_{0} P_{0}=\frac{1}{3} N \epsilon_{0}(\gamma) I^{(1)}
$$

In this formula $I^{(1)}$ is the unit operator in the said space of dimension $2^{N / 3}$. Now we consider a transformed projection operator

$$
P_{1}(a)=u(a) P_{0} u(a)^{+}
$$

and the corresponding projection of the total Hamiltonian $H$

$$
P_{1}(a) H P_{1}(a)=u(a) P_{0} u(a)^{+} H u(a) P_{0} u(a)^{+}
$$

Instead of $(10)$ we consider the projected operator

$$
P_{0} u(a)^{\dagger} H u(a) P_{0}=H^{(1)}\left(\mathbf{S}_{i}^{(1)}, a\right)
$$

which has the same eigenvalues as (10). In this paper we are only interested in an upper bound for the lowest eigenvalue of $H$ and it is easily seen that such an upper bound is found in the lowest eigenvalue of $H^{(1)}$. More generally an upper bound for this last eigenvalue will also limit the lowest eigenvalue of $H$. It turns out that in our special example $H^{(1)}$ is a reproduction of $H$ in terms of cell spins defined below, apart from an additive and a multiplicative constant:

$$
\begin{gathered}
H^{(1)}\left(\mathbf{S}_{i}^{(1)}, a\right)=\epsilon_{0}(a, \gamma) N I^{(1)}+c_{0}(a, \gamma) H\left(\mathbf{S}_{i}^{(1)}, \gamma^{(1)}(a, \gamma)\right) \\
\left\langle\frac{1}{2}+, m_{i}^{\prime}\left|S_{i 2}^{(1)}\right| \frac{1}{2}+, m_{i}\right\rangle=m_{i} \delta_{m_{i}^{\prime}, m_{i}} \\
\left\langle\frac{1}{2}+, m_{i}{ }^{\prime}\left|S_{i \pm}^{(1 \pm}\right| \frac{1}{2}+, m_{i}\right\rangle=\delta_{m_{i}^{\prime}, m_{i} \pm 1}
\end{gathered}
$$


The first step in our calculations is the determination of $u(a)^{+} H u(a)$, for which we start with the transformed operators corresponding to the spins $\mathbf{S}_{3 k \pm 1}, \mathbf{S}_{3 k}$ of one cell. These are easily calculated on the basis of (4) and (5):

$$
\begin{aligned}
u(a)^{+} \mathbf{S}_{3 k} u(a)= & \mathbf{S}_{3 k} \\
u(a)^{+} \mathbf{S}_{3 k+1} u(a)= & \cos ^{2} a \mathbf{S}_{3 k+1}+\sin ^{2} a \mathbf{S}_{3 k+2} \\
& +2 \cos a \sin a\left(\mathbf{S}_{3 k+1} \times \mathbf{S}_{3 k+2}\right) \\
u(a)^{+} \mathbf{S}_{3 k-1} u(a)= & \cos ^{2} a \mathbf{S}_{3 k-1}+\sin ^{2} a \mathbf{S}_{3 k-2} \\
& -2 \cos a \sin a\left(\mathbf{S}_{3 k-2} \times \mathbf{S}_{3 k-1}\right)
\end{aligned}
$$

From the relations (14) the transformations of the different parts of $H$ introduced in (1) follow immediately

$$
\begin{aligned}
u(a)^{+} & H_{0, k} u(a) \\
= & 4\left\{\left[\cos ^{2} a \mathbf{S}_{3 k-1}+\sin ^{2} a \mathbf{S}_{3 k-2}-2 \cos a \sin a\left(\mathbf{S}_{3 k-2} \times \mathbf{S}_{3 k-1}\right)\right] \cdot \mathbf{S}_{3 k}\right. \\
& +\mathbf{S}_{3 k} \cdot\left[\cos ^{2} a \mathbf{S}_{3 k+1}+\sin ^{2} a \mathbf{S}_{3 k+2}+2 \cos a \sin a\left(\mathbf{S}_{3 k+1} \times \mathbf{S}_{3 k+2}\right)\right] \\
& +\gamma\left[\cos ^{2} a \mathbf{S}_{3 k-1}+\sin ^{2} a \mathbf{S}_{3 k-2}-2 \cos a \sin a\left(\mathbf{S}_{3 k-2} \times \mathbf{S}_{3 k-1}\right)\right] \\
& \left.\times\left[\cos ^{2} a \mathbf{S}_{3 k+1}+\sin ^{2} a \mathbf{S}_{3 k+2}+2 \cos a \sin a\left(\mathbf{S}_{3 k+1} \times \mathbf{S}_{3 k+2}\right)\right]\right\}
\end{aligned}
$$

$$
\begin{aligned}
& u(a)^{+} H_{k, k+1}^{\prime} u(a) \\
&= 4\left(\mathbf{S}_{3 k+1} \cdot \mathbf{S}_{3 k+2}+\gamma\left\{\mathbf { S } _ { 3 k } \cdot \left[\cos ^{2} a \mathbf{S}_{3 k+2}+\sin ^{2} a \mathbf{S}_{3 k+1}\right.\right.\right. \\
&\left.-2 \cos a \sin a\left(\mathbf{S}_{3 k+1} \times \mathbf{S}_{3 k+2}\right)\right] \\
&+\left[\cos ^{2} a \mathbf{S}_{3 k+1}+\sin ^{2} a \mathbf{S}_{3 k+2}\right. \\
&\left.\left.\left.+2 \cos a \sin a\left(\mathbf{S}_{3 k+1} \times \mathbf{S}_{3 k+2}\right)\right] \cdot \mathbf{S}_{3 k+3}\right\}\right)
\end{aligned}
$$

The next step in our analysis is the projection of the transformed parts of the total Hamiltonian and for this projection we need expressions for the projected operators for the individual spins and the dyadic forms in terms of two spins of the same cell. These projections were given already in I and they are reproduced here in Table I. Now the projections of the transformed operators given in (15) and (16) are easily determined and from this projection the Hamiltonian $H^{(1)}$ for the scaled system follows immediately. The projec-

Table I. Projected Operators for Cell $k$

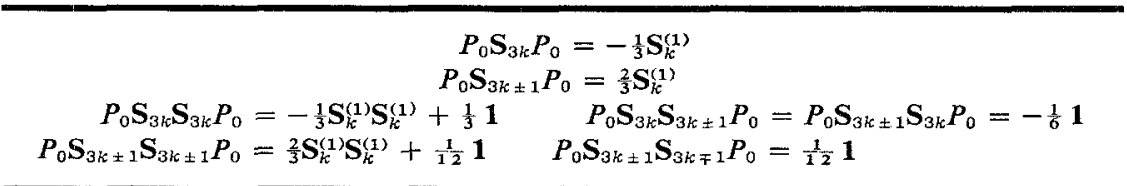


tions of (15) and (16), respectively, are

$$
\begin{aligned}
P_{0} u(a)^{+} & H_{0, k} u(a) P_{0} \\
= & 4\left\{-\cos ^{2} a-\frac{2}{9} \sin ^{2} a\left(\mathbf{S}_{k-1}^{(1)} \cdot \mathbf{S}_{k}^{(1)}+\mathbf{S}_{k}^{(1)} \cdot \mathbf{S}_{k+1}^{(1)}\right)\right. \\
& +\gamma\left[\frac{1}{4} \cos ^{4} a+\frac{4}{9} \cos ^{2} a \sin ^{2} a\left(\mathbf{S}_{k-1}^{(1)} \cdot \mathbf{S}_{k}^{(1)}+\mathbf{S}_{k}^{(1)} \cdot \mathbf{S}_{k+1}^{(1)}\right)\right. \\
& \left.\left.+\left(\frac{4}{9} \sin ^{4} a+(8 / 27) \cos ^{2} a \sin ^{2} a\right) \mathbf{S}_{k-1}^{(1)} \cdot \mathbf{S}_{k+1}^{(1)}\right]\right\} \\
P_{0} u(a)+ & H_{k, k+1}^{\prime} u(a) P_{0} \\
= & 4\left[{ }_{9}^{4} \mathbf{S}_{k}^{(1)} \cdot \mathbf{S}_{k+1}^{(1)}+\gamma\left(-\sin ^{2} a-\frac{4}{9} \cos ^{2} a \mathbf{S}_{k}^{(1)} \cdot \mathbf{S}_{k+1}^{(1)}\right)\right]
\end{aligned}
$$

and the Hamiltonian $H^{(1)}$ introduced in (12) turns out to be

$$
\begin{aligned}
H^{(1)}\left(\mathbf{S}_{i}^{(1)}, a\right)= & -\frac{4}{3}\left[\gamma+(1-\gamma) x-\frac{1}{4} \gamma x^{2}\right] N I^{(1)} \\
& +\frac{4}{9} x[(1+\gamma)-2 \gamma x] 4 \sum_{i} \mathbf{S}_{i}^{(1)} \cdot \mathbf{S}_{i+1}^{(1)} \\
& +(4 / 27) \gamma(1-x)(3-x) 4 \sum_{i} \mathbf{S}_{i}^{(1)} \cdot \mathbf{S}_{i+2}^{(1)}, \quad x=\cos ^{2} a
\end{aligned}
$$

This Hamiltonian is a reproduction of the original one, in the sense that its variables are the cell-spin operators for the lowest Kramers doublets. Again we have an example of a Kadanoff scaling ${ }^{(8)}$ with cells of three spins. ${ }^{(1)}$ The scaling is characterized by three functions $\epsilon_{0}, c_{0}$, and $\gamma^{(1)}$ defined by formula (12). Explicit expressions for these functions are

$\epsilon_{0}(x, \gamma)=-\frac{4}{3}\left[\gamma+(1-\gamma) x-\frac{1}{4} \gamma x^{2}\right], \quad c_{0}(x, \gamma)=\frac{4}{9} x[(1+\gamma)-2 \gamma x]$

$\gamma^{(1)}(x, \gamma)=\frac{1}{3} \gamma(1-x)(3-x) / x[(1+\gamma)-2 \gamma x], \quad x=\cos ^{2} a$

In (19) and (20) we have introduced the new variable $x$, which gives an important simplification of the formulas. We still have the freedom to choose for $x$ any value between zero and one $[a$ is real as a consequence of the $u(a)$ being unitary]. In the next section it is shown how the optimal value for $x$ is chosen, in order to get the best value for the upper bound of the lowest energy per spin.

\section{EQUATION FOR THE UPPER BOUND OF THE GROUND-STATE ENERGY}

As was already stated in the previous section, the lowest eigenvalue of $H$ has an upper bound given by the lowest eigenvalue of $H^{(1)}$. Introducing the symbols $\epsilon(\gamma)$ and $\epsilon^{(1)}(x, \gamma)$ for the respective energies per spin, we have the inequality

$$
\epsilon(\gamma) \leqslant \epsilon^{(1)}(x, \gamma)=\epsilon_{0}(x, \gamma)+\frac{1}{3} c_{0}(x, \gamma) \epsilon\left(\gamma^{(1)}(x, \gamma)\right)
$$

as follows from (12). The factor $1 / 3$ is a consequence of the fact that the number of cells is $1 / 3$ of the total number of spins in our special case. For the case $c_{0}(x, \gamma) \geqslant 0$ the inequality (21) may be used an unlimited number 
of times, which results in a series in terms of an infinite number of parameters $x^{(s)}, s=0,1,2,3, \ldots$.We make the supposition that this series converges in a neighborhood of the set of values for $x^{(s)}$ that corresponds to the optimal value for the series giving the lowest upper bound for $\epsilon(\gamma)$.

Under this condition we may write

$$
\begin{aligned}
\epsilon(\gamma) \leqslant & \epsilon^{(1)}(x, \gamma) \\
\leqslant & \epsilon_{0}(x, \gamma)+\frac{1}{3} c_{0}(x, \gamma) \epsilon_{0}\left(x^{(1)}, \gamma^{(1)}(x, \gamma)\right) \\
& +\frac{1}{9} c_{0}(x, \gamma) c_{0}\left(x^{(1)}, \gamma^{(1)}(x, \gamma)\right) \\
& \times \epsilon_{0}\left(x^{(2)}, \gamma^{(1)}\left(x^{(1)}, \gamma^{(1)}(x, \gamma)\right)+\cdots\right. \\
= & \epsilon_{0}(x, \gamma)+\sum_{s=1}^{\infty}\left(1 / 3^{s}\right)\left[\prod_{t=0}^{s-1} c_{0}\left(x^{(t)}, \gamma^{(t)}\right)\right] \epsilon_{0}\left(x^{(s)}, \gamma^{(s)}\right) \\
& \gamma^{(t)}=\gamma^{(1)}\left(x^{(t-1)}, \gamma^{(t-1)}\right), \quad t=1,2,3, \ldots \\
x^{(0)}= & x, \quad \gamma^{(0)}=\gamma ; \quad 0 \leqslant x^{(i)} \leqslant 1, \quad i=0,1,2, \ldots
\end{aligned}
$$

Defining $\epsilon^{*}(\gamma)$ as the minimum value for the series in (22), we have

$\epsilon \leqslant \epsilon^{*}(\gamma)=\operatorname{Min}_{x, x^{(i)}}\left\{\epsilon_{0}(x, \gamma)+\sum_{s=1}^{\infty} \frac{1}{3^{s}}\left[\prod_{t=0}^{s-1} c_{0}\left(x^{(t)}, \gamma^{(t)}\right)\right] \epsilon_{0}\left(x^{(s)}, \gamma^{(s)}\right)\right\}$

Now we make the hypothesis that for this minimum $c_{0}\left(x^{(t)}, \gamma^{(t)}\right) \geqslant 0$, which enables us to write (23) in a compact form

$\epsilon^{*}(\gamma)=\operatorname{Min}_{x}\left[\epsilon_{0}(x, \gamma)+\frac{1}{3} c_{0}(x, \gamma) \epsilon^{*}\left(\gamma^{(1)}(x, \gamma)\right)\right], \quad c_{0}(x, \gamma) \geqslant 0$

Formula (24) certainly gives an upper bound for $\epsilon$, but it is not certain that this is identical with the solution of (23). We come back to this point at the end of Section 5. The solution of (24) is independent of the condition $c_{0}(x, \gamma)$ $\geqslant 0$. The three functions $\epsilon_{0}, c_{0}$, and $\gamma^{(1)}$ are given by (20). Now the determination of the lowest upper bound for the energy per spin is reduced to a mathematical problem, the solution of which is given in the next section.

\section{THE UPPER BOUND OF THE GROUND-STATE ENERGY PER SPIN}

A first step to the solution of (24) was made in a purely analytical way. It is easily shown that $\gamma^{(1)}(x, 0)=0(x \neq 0)$ and excluding the value $x=0$, we find the following equation for $\epsilon^{*}(0)$ :

$$
\epsilon^{*}(0)=\operatorname{Min}_{x \neq 0}\left[\epsilon_{0}(x, 0)+\frac{1}{3} c_{0}(x, 0) \epsilon^{*}(0)\right]
$$

which can be solved in an elementary way to give

$$
\epsilon^{*}(0)=-36 / 23, \quad \hat{x}(0)=1
$$


Table II. Analytical Solution for $\epsilon^{*}(\gamma)$ [Eq. (24)]

$$
\begin{aligned}
-\infty<\gamma<\gamma_{1}=\frac{1458}{2459} & \epsilon^{*}(\gamma)=-\frac{36}{23}+\frac{13}{23} \gamma & \hat{x}(\gamma)=1 \\
\gamma_{1}<\gamma<\gamma_{2} & \epsilon^{*}(\gamma)=-\frac{26244}{35351} \frac{1}{\gamma}+\frac{33192}{35351}-\frac{4428220}{2863431} \gamma & \hat{x}(\gamma)=\frac{1458-922 \gamma}{1537 \gamma} \\
\gamma_{2}<\gamma<\gamma_{3}=\frac{69}{85} & \epsilon^{*}(\gamma)=-\frac{19044}{10971} \frac{1}{\gamma}+\frac{46920}{10971}-\frac{46072}{10971} \gamma & \hat{x}(\gamma)=\frac{138-170 \gamma}{53 \gamma} \\
\gamma_{3}<\gamma<\infty & \epsilon^{*}(\gamma)=-\frac{36}{23} \gamma & \hat{x}(\gamma)=0
\end{aligned}
$$

$\hat{x}(0)$ being the value for $x$ corresponding to the minimum of the right-hand side of (25). In general the minimum in (24) will correspond to a value of $x$ being a function of $\gamma: \hat{x}(\gamma)$. For $\gamma=0$ there is a boundary extremum and making the hypothesis $\hat{x}(\gamma) \equiv 1$ in a finite interval with $\gamma=0$ as an interior point, we find the solution

$$
\epsilon^{*}(\gamma)=\epsilon_{0}(1, \gamma)+\frac{1}{3} c_{0}(1, \gamma) \epsilon^{*}(0)=-(36 / 23)+(13 / 23) \gamma
$$

in the neighborhood of $\gamma=0$. We took (27) as a zeroth-order solution for all (real) values of $\gamma$ and solved (24) numerically with an iteration procedure,

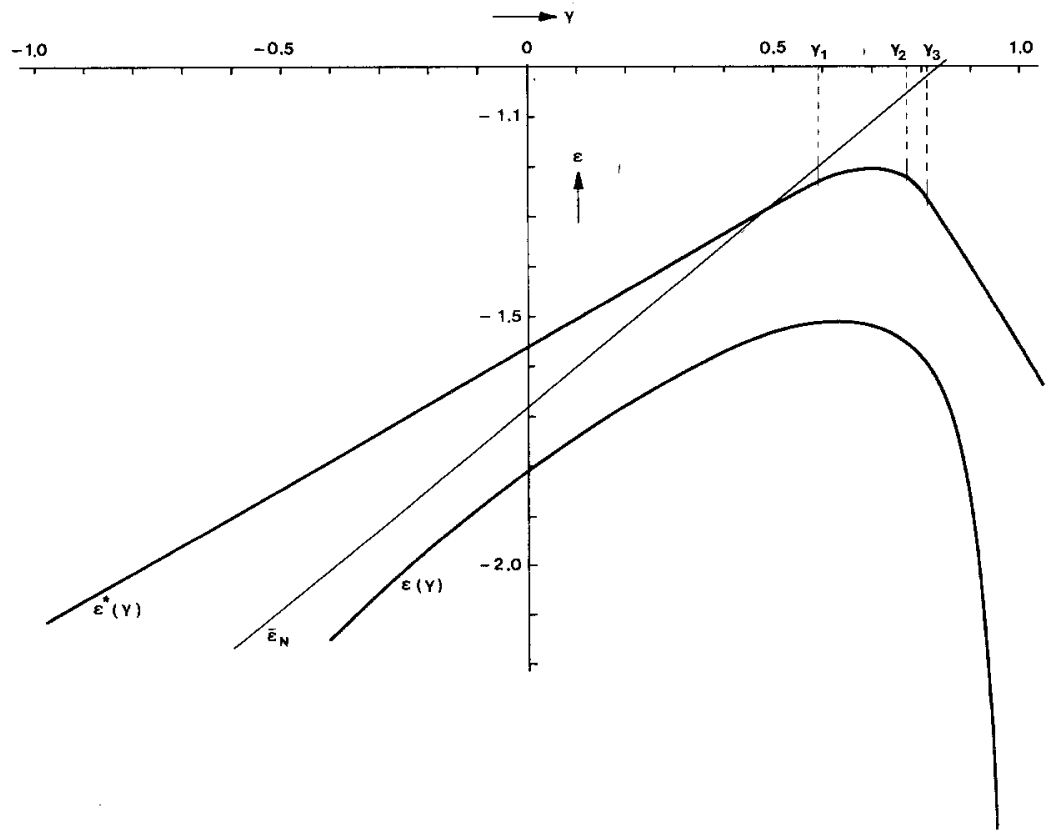

Fig. 1. Energy per spin for the linear chain. 
Table III. $\epsilon^{*}$ as a Function of $\gamma$

\begin{tabular}{|c|c|c|c|}
\hline$\gamma$ & $\epsilon^{*}$ & $\gamma$ & $\epsilon^{*}$ \\
\hline-1.00 & -2.13043 & 0.65 & -1.20841 \\
\hline-0.95 & -2.10217 & 0.66 & -1.20657 \\
\hline-0.90 & -2.07391 & 0.67 & -1.20525 \\
\hline-0.85 & -2.04565 & 0.68 & -1.20442 \\
\hline-0.80 & -2.01739 & 0.69 & -1.20406 \\
\hline-0.75 & -1.98913 & 0.70 & -1.20415 \\
\hline-0.70 & -1.96087 & 0.71 & -1.20468 \\
\hline-0.65 & -1.93261 & 0.72 & -1.20562 \\
\hline-0.60 & -1.90435 & 0.73 & -1.20696 \\
\hline-0.55 & -1.87609 & 0.74 & -1.20868 \\
\hline-0.50 & -1.84783 & 0.75 & -1.21077 \\
\hline-0.45 & -1.81957 & 0.76 & -1.21321 \\
\hline-0.40 & -1.79130 & 0.77 & -1.21599 \\
\hline-0.35 & -1.76304 & $\gamma_{2}=0.77487$ & -1.21746 \\
\hline-0.30 & -1.73478 & 0.78 & -1.22428 \\
\hline-0.25 & -1.70652 & 0.79 & -1.23810 \\
\hline-0.20 & -1.67826 & 0.80 & -1.25263 \\
\hline-0.15 & -1.65000 & 0.81 & -1.26783 \\
\hline-0.10 & -1.62174 & $\gamma_{3}=69 / 85$ & -1.27059 \\
\hline-0.05 & -1.59348 & 0.82 & -1.28348 \\
\hline 0 & -1.56522 & 0.83 & -1.29913 \\
\hline 0.05 & -1.53696 & 0.84 & -1.31478 \\
\hline 0.10 & -1.50870 & 0.85 & -1.33043 \\
\hline 0.15 & -1.48043 & 0.86 & -1.34609 \\
\hline 0.20 & -1.45217 & 0.87 & -1.36174 \\
\hline 0.25 & -1.42391 & 0.88 & -1.37739 \\
\hline 0.30 & -1.39565 & 0.89 & -1.39304 \\
\hline 0.35 & -1.36739 & 0.90 & -1.40870 \\
\hline 0.40 & -1.33913 & 0.91 & -1.42435 \\
\hline 0.45 & -1.31087 & 0.92 & -1.44000 \\
\hline 0.50 & -1.28261 & 0.93 & -1.45565 \\
\hline 0.55 & -1.25435 & 0.94 & -1.47130 \\
\hline$\gamma_{1}=1458 / 2459$ & -1.23009 & 0.95 & -1.48696 \\
\hline 0.60 & -1.22626 & 0.96 & -1.50261 \\
\hline 0.61 & -1.22144 & 0.97 & -1.51826 \\
\hline 0.62 & -1.21728 & 0.98 & -1.53391 \\
\hline 0.63 & -1.21374 & 0.99 & -1.54957 \\
\hline 0.64 & -1.21079 & 1.00 & -1.56522 \\
\hline
\end{tabular}

performed on a DEC10 computer. The numerical solution gave for $\gamma \geqslant 0.82$ a boundary extremum $\hat{x}(\gamma)=0$. Using this extra information together with the favorable mapping property of $\gamma^{(1)}(\hat{x}(\gamma), \gamma)$ (see below), it was possible to construct an analytical solution, which is given in Table II.

The continuous curve consists of two parts represented by straight 
lines and two hyperbolic parts. The values $\gamma_{1}, \gamma_{2}$, and $\gamma_{3}$ correspond to the intersection of two adjacent parts, $\gamma_{2}$ being the largest root of the quadratic equation

$$
1,899,143 \gamma^{2}-2,389,392 \gamma+711,180=0, \quad \gamma_{2}=0.77487
$$

The function $\hat{x}(\gamma)$ is continuous, with the exception of the point $\gamma=\gamma_{2}$, for which there is a finite discontinuity. The curve $\epsilon^{*}(\gamma)$ is shown in Fig. 1 .

Having found the analytical solution, it is easy to prove that this solution obeys (24) in such a sense that for each of the four parts of the curve, $\hat{x}(\gamma)$ corresponds to a local minimum, which is a boundary extremum for $\gamma<\gamma_{1}$ and $\gamma>\gamma_{3}$. The function $\gamma^{(1)}(\hat{x}(\gamma), \gamma)$ maps the interval $\left[\gamma_{1}, \gamma_{2}\right]$ on an interval $\left[0, \bar{\gamma}_{2}\right]$ which is part of $\left[0, \gamma_{1}\right]$, whereas it maps $\left[\gamma_{2}, \gamma_{8}\right]$ on $\left[\bar{\gamma}_{2}, \infty\right]$, the latter being part of $\left[\gamma_{3}, \infty\right]$. In Table III we give numerical values for $\epsilon^{*}$ based on the formulas of Table II. The comparison of our values with former results strongly suggests that the restriction $c_{0}\left(x^{(t)}, \gamma^{(t)}\right) \geqslant 0$ made in Section 4 can be omitted.

\section{CONCLUDING REMARKS}

In Fig. 1 we have also plotted some former results for the same problem. We show the upper bound calculated by Niemeijer ${ }^{(4)}$

$$
\bar{\epsilon}_{N}=4\left(-\frac{1}{\pi}-\frac{1}{\pi^{2}}+\frac{2 \gamma}{\pi^{2}}\right)=-1.6785+0.8106 \gamma
$$

and also the curve $\epsilon(\gamma)$ corresponding to the perturbation calculation of I.

We should mention the exact result for $\gamma=0$, given by Hulthén ${ }^{(5)}$

$$
\epsilon_{H}(0)=1-4 \log 2=-1.7726
$$

For a survey of the results for the linear chain we refer to a paper by Thompson. ${ }^{(6)}$ For the sake of clarity we have not drawn the results of Majumdar and Ghosh ${ }^{(7)}$ for a chain of ten spins. These results were compared with $\epsilon(\gamma)$ in our previous paper. ${ }^{(1)}$

It is clear that $\epsilon^{*}(\gamma)$ gives an improved upper bound as compared with Niemeijer's results for $\gamma \geqslant 0.5$. It also shows the same global dependence on $\gamma$ as $\epsilon(\gamma)$ and the curve of Majumdar and Ghosh. We further believe that for $\gamma \rightarrow 1$ the curve for $\epsilon(\gamma)$ is below the exact result, because $\epsilon(\gamma)$ diverges to $-\infty$ for $\gamma \rightarrow 1$.

\section{ACKNOWLEDGMENT}

The authors are indebted to Dr. F. W. Wiegel for his stimulating interest in this work and for a critical reading of the manuscript. 


\section{REFERENCES}

1. H. P. van de Braak, W. J. Caspers, C. de Lange, and M. W. M. Willemse, Physica 87A: 354 (1977).

2. H. P. van de Braak, W. J. Caspers, C. de Lange, and M. W. M. Willemse, in Annals of the Israel Physical Society, Vol. 2, Proceedings of the 13th IUPAP Conference on Statistical Physics, Haifa 1977 (Adam Hilger, Bristol and The Israel Physical Society, in association with The American Institute of Physics, New York, 1978); H. P. van de Braak, W. J. Caspers, and M. W. M. Willemse, Phys. Lett. 67A:147 (1978); H. P. van de Braak, W. J. Caspers, F. W. Wiegel, and M. W. M. Willemse, J. Stat. Phys. 18: 577 (1978).

3. J. H. van Vleck, The Theory of Electric and Magnetic Susceptibilities (Oxford University Press, 1932), p. 316; W. J. Caspers, Physics 1:45 (1964).

4. Th. Niemeijer, J. Math. Phys. 12:1487 (1971).

5. L. Hulthén, Ark. Mat. Astron. Fysik 26A, No. 11 (1938).

6. C. J. Thompson, in Phase Transitions and Critical Phenomena, Vol. I, C. Domb and M. S. Green, eds. (Academic Press, London, New York, 1972), p. 177.

7. C. K. Majumdar and D. K. Ghosh, J. Math. Phys. 10:1388, 1399 (1969).

8. L. P. Kadanoff, Physics 2:263 (1966); B. Widom, in Fundamental Problems in Statistical Mechanics, Vol. III, Proceedings of the 1974 Wageningen Summer School, E. G. D. Cohen, ed. (North-Holland, Amsterdam, 1975), p. 1. 